\title{
Dual-wavelength excited photoluminescence spectroscopy of deep-level hole traps in Ga(In)NP
}

Daniel Dagnelund, Yuqing Huang, C. W. Tu, H. Yonezu, Irina Buyanova and Weimin Chen

\author{
Linköping University Post Print
}

\section{Tweet}

N.B.: When citing this work, cite the original article.

Original Publication:

Daniel Dagnelund, Yuqing Huang, C. W. Tu, H. Yonezu, Irina Buyanova and Weimin Chen, Dual-wavelength excited photoluminescence spectroscopy of deep-level hole traps in Ga(In)NP, 2015, Journal of Applied Physics, (117), 015701.

http://dx.doi.org/10.1063/1.4905274

Copyright: American Institute of Physics (AIP) http://www.aip.org/

Postprint available at: Linköping University Electronic Press http://urn.kb.se/resolve?urn=urn:nbn:se:liu:diva-113961 


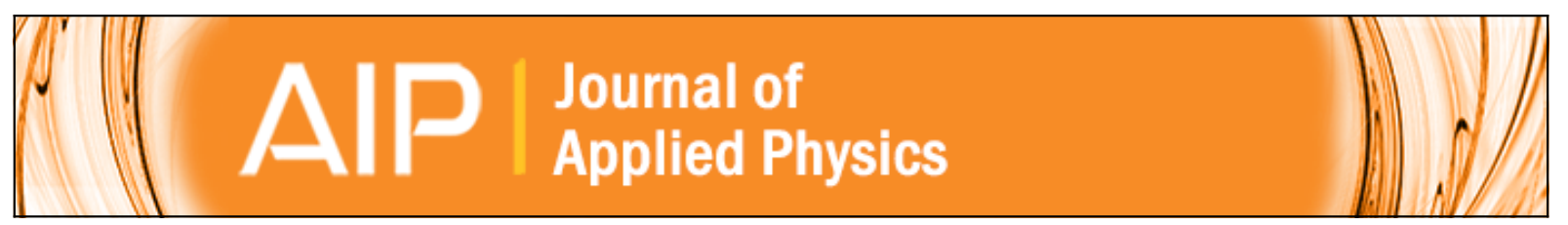

Dual-wavelength excited photoluminescence spectroscopy of deep-level hole traps in Ga(In)NP

D. Dagnelund, Y. Q. Huang, C. W. Tu, H. Yonezu, I. A. Buyanova, and W. M. Chen

Citation: Journal of Applied Physics 117, 015701 (2015); doi: 10.1063/1.4905274

View online: http://dx.doi.org/10.1063/1.4905274

View Table of Contents: http://scitation.aip.org/content/aip/journal/jap/117/1?ver=pdfcov

Published by the AIP Publishing

\section{Articles you may be interested in}

Deep hole traps in undoped $n-G a N$ films grown by hydride vapor phase epitaxy

J. Appl. Phys. 115, 223702 (2014); 10.1063/1.4882715

Crystal-field split levels of $\mathrm{Nd} 3$ + ions in GaN measured by luminescence spectroscopy

J. Appl. Phys. 105, 053101 (2009); 10.1063/1.3082500

Acceptor levels in GaSe:In crystals investigated by deep-level transient spectroscopy and photoluminescence J. Appl. Phys. 103, 013710 (2008); 10.1063/1.2831130

Origin and annealing of deep-level defects in $\mathrm{p}$-type $\mathrm{GaAs} / \mathrm{Ga}(\mathrm{As}, \mathrm{N}) / \mathrm{GaAs}$ heterostructures grown by molecular beam epitaxy

J. Appl. Phys. 89, 6294 (2001); 10.1063/1.1370115

Optically and thermally detected deep levels in n-type Schottky and $p+-n$ GaN diodes

Appl. Phys. Lett. 76, 3064 (2000); 10.1063/1.126580

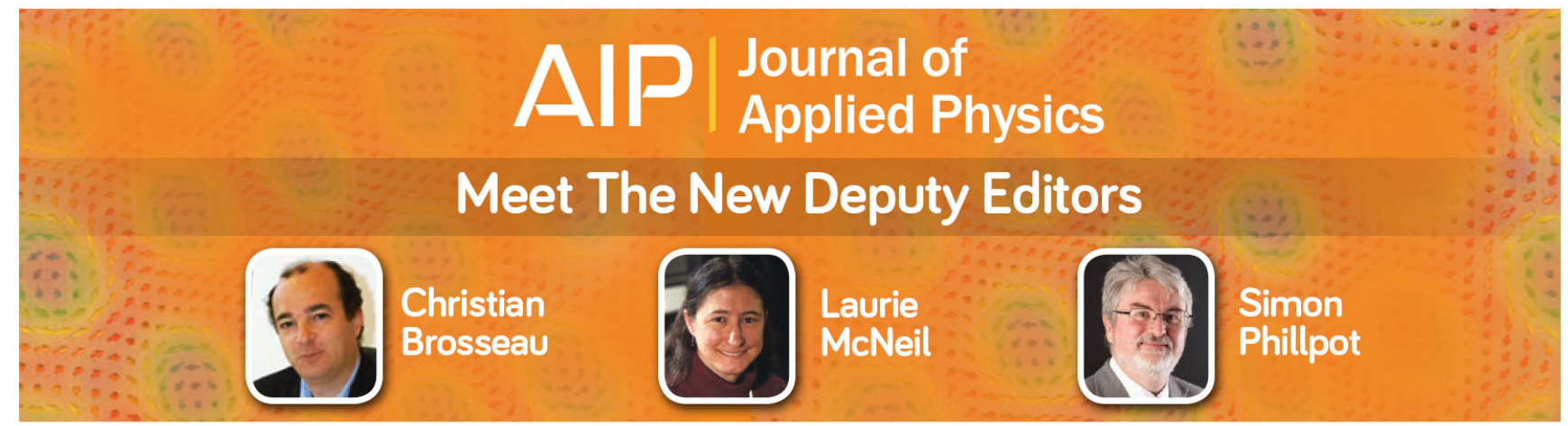




\title{
Dual-wavelength excited photoluminescence spectroscopy of deep-level hole traps in $\mathbf{G a}(\mathbf{I n}) \mathbf{N P}$
}

\author{
D. Dagnelund, ${ }^{1}$ Y. Q. Huang, ${ }^{1}$ C. W. Tu, ${ }^{2}$ H. Yonezu, ${ }^{3}$ I. A. Buyanova, ${ }^{1}$ and W. M. Chen ${ }^{1}$ \\ ${ }^{1}$ Department of Physics, Chemistry and Biology, Linköping University, S-581 83 Linköping, Sweden \\ ${ }^{2}$ Department of Electrical and Computer Engineering, University of California, La Jolla, CA 92093, USA \\ ${ }^{3}$ Department of Electrical and Electronic Engineering, Toyohashi University of Technology, Toyohashi, \\ Aichi 441-8580, Japan
}

(Received 2 October 2014; accepted 18 December 2014; published online 5 January 2015)

\begin{abstract}
By employing photoluminescence (PL) spectroscopy under dual-wavelength optical excitation, we uncover the presence of deep-level hole traps in $\mathrm{Ga}(\mathrm{In}) \mathrm{NP}$ alloys grown by molecular beam epitaxy (MBE). The energy level positions of the traps are determined to be at $0.56 \mathrm{eV}$ and $0.78 \mathrm{eV}$ above the top of the valance band. We show that photo-excitation of the holes from the traps, by a secondary light source with a photon energy below the bandgap energy, can lead to a strong enhancement (up to $25 \%$ ) of the PL emissions from the alloys under a primary optical excitation above the bandgap energy. We further demonstrate that the same hole traps can be found in various MBEgrown $\mathrm{Ga}(\mathrm{In}) \mathrm{NP}$ alloys, regardless of their growth temperatures, chemical compositions, and strain. The extent of the PL enhancement induced by the hole de-trapping is shown to vary between different alloys, however, likely reflecting their different trap concentrations. The absence of theses traps in the GaNP alloy grown by vapor phase epitaxy suggests that their incorporation could be associated with a contaminant accompanied by the $\mathrm{N}$ plasma source employed in the MBE growth, possibly a Cu impurity. @ 2015 AIP Publishing LLC. [http://dx.doi.org/10.1063/1.4905274]
\end{abstract}

\section{INTRODUCTION}

Alloying of gallium phosphide $(\mathrm{GaP})$ with nitrogen $(\mathrm{N})$ leads to a giant bandgap bowing ${ }^{1-4}$ and $\mathrm{N}$-induced transformation from an indirect to a quasi-direct bandgap, ${ }^{4,5}$ largely improving radiative efficiency. Further alloying with In, resulting in GaInNP, adds additional freedom in bandgap and strain engineering desirable in exploring hetero- and quantumstructures for high-performance devices. This makes the $\mathrm{Ga}(\mathrm{In}) \mathrm{NP}$ alloys attractive for optoelectronic applications, ${ }^{1,2}$ such as in light-emitting devices that cover the amber spectral range and novel intermediate-band solar cells ${ }^{6,7}$ The fact that GaNP can be lattice matched to Si further extends the range of potential applications that will integrate superior optoelectronic properties of III-V semiconductors with the mainstream Si microelectronics. Before the full potential of this materials system can be realized, however, a number of key material and physical properties need to be addressed. One of the most important problems is defects (including impurities), which are more profound in this class of highly mismatched materials due to the large disparity in the size and electronegativity between the anion atoms of the alloys as well as the required non-equilibrium growth conditions. Defects in these dilute nitrides are known to often act as efficient non-radiative recombination centers and carrier traps, severely deteriorating optical and transport properties of the materials and hindering them from practical applications. ${ }^{8-11}$ On the other hand, extraordinary room-temperature spin functionalities promising for spintronic applications were also demonstrated by spin engineering of certain defects in $\mathrm{Ga}(\mathrm{In}) \mathrm{NAs}$ alloys ${ }^{12-16}$ - another family of dilute nitrides. A present challenge in development of high-quality $\mathrm{Ga}(\mathrm{In}) \mathrm{NP}$ alloys lies in understanding and control of defects introduced during growth. Previous studies using the optically detected magnetic resonance (ODMR) technique have identified $\mathrm{Ga}$ interstitials commonly found in $\mathrm{Ga}(\mathrm{In}, \mathrm{Al}) \mathrm{NP}^{9,17-22}$ and related GaNAsP (Ref. 23) and $\mathrm{Ga}(\mathrm{In}, \mathrm{Al}) \mathrm{NAs}$ alloys, ${ }^{10,24-26}$ as well as P-related interfacial/ surface defects in GaNP/GaP heterostructure ${ }^{27}$ and core/shell nanowires (NWs), ${ }^{28}$ as important defects in carrier recombination that can severely limit efficiencies of optoelectronic and photovoltaic devices. As to carrier traps that are also important in device applications of $\mathrm{Ga}(\mathrm{In}) \mathrm{NP}$, very little is known so far. ${ }^{11}$

In this work, we carried out a comprehensive and systematic study of defects in the Ga(In)NP alloys that act as carrier traps and can affect light-emitting efficiency. This was done by means of purely optical and nondestructive techniques of dual-wavelength excited photoluminescence (PL) (DEPL), DEPL excitation, and time-resolved DEPL. These methods are especially suited for detection of carrier traps that are important in light emissions and carrier extraction. This is because PL under a primary optical excitation above the bandgap energy (denoted by P1 below) is directly monitored, see Fig. 1, while a secondary light with a photon energy below the bandgap (denoted by P2 below) excites holes (or electrons) from the traps to the valence band (VB) (or the conduction band (CB)). In this way, only those traps with a significant impact in free carrier concentrations and thus PL are selected in the study.

\section{SAMPLES AND METHODS}

Eight $\mathrm{Ga}(\mathrm{In}) \mathrm{NP} / \mathrm{GaP}$ structures grown on (001) $\mathrm{GaP}$ substrates and two GaNP/Si structures grown on $\mathrm{Si}$ substrates were chosen for this study. They were grown by gassource or solid-source molecular beam epitaxy, denoted by 


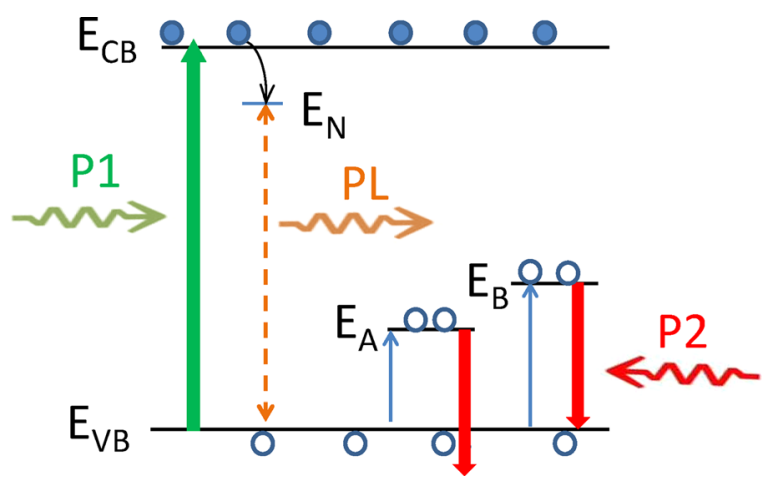

FIG. 1. A schematic illustration of the proposed model for the hole trapping and NIR-induced hole de-trapping processes, marked by the thin blue arrows and the thick red arrows, respectively. $\mathrm{E}_{\mathrm{N}}$ denotes the $\mathrm{N}$-related localized states that give rise to the PL emissions monitored in this study. $\mathrm{E}_{\mathrm{A}}$ and $\mathrm{E}_{\mathrm{B}}$ represent two hole trap levels with different energy positions within the bandgap. $\mathrm{E}_{\mathrm{CB}}$ and $\mathrm{E}_{\mathrm{VB}}$ denote the conduction band and valence band edge, respectively.

GS-MBE or SS-MBE, using a plasma-assisted nitrogen source. The most important growth parameters of the structures are summarized in Table I. The $\mathrm{N}$ compositions of the alloys were determined by high resolution X-ray diffraction measurements. The studied structures can be grouped into five pairs, with each having a specific aim. The first pair comprised two GaNP epilayers with a large difference (28 times) in $\mathrm{N}$ concentration, employed to study the effect of $\mathrm{N}$. The second pair was obtained after the first pair was treated by post-growth hydrogenation with a Kaufmann source at $300^{\circ} \mathrm{C}$, using a low $\mathrm{H}$ ion energy $(100 \mathrm{eV})$ and with a dose of $[\mathrm{H}]=1 \times 10^{18} \mathrm{~cm}^{-2}$. Details of the hydrogenation can be found elsewhere. ${ }^{26}$ For the third and fourth pairs, the focus was on alloying with In and doping by shallow donors and acceptors, respectively. The fifth pair of samples consists of a GaNP epilayer grown on a (001) Si substrate and GaNP/ $\mathrm{GaP}$ shell/core NWs grown on a (111) $\mathrm{Si}$ substrate in order to study trap formation in the GaNP alloys grown on $\mathrm{Si}$. A detailed description of the NW growth process can be found in Refs. 29 and 30. To examine whether or not the introduction of the studied carrier traps was directly promoted by the
MBE growth, we also studied an undoped GaNP epilayer grown by vapor phase epitaxy (VPE) as a reference sample. The details of the VPE growth can be found in Ref. 23 .

DEPL measurements were performed using either the $532 \mathrm{~nm}$ of a solid-state laser or the $632.8 \mathrm{~nm}$ line of a He-Ne laser as the primary excitation source, with a photon energy above the bandgap energy of the studied $\mathrm{Ga}$ (In)NP alloys. One of the following three near-infrared (NIR) light sources was used for the secondary excitation: the $870-950 \mathrm{~nm}$ line from a Ti:Sapphire laser, the $915 \mathrm{~nm}$ line from a solid-state laser diode, or $800-2400 \mathrm{~nm}$ light from a tungsten halogen lamp which was dispersed through a 0.2 -m single grating monochromator (used in DEPL excitation measurements). Laser spots focused onto the sample were typically less than $0.5 \mathrm{~mm}$ in diameter. PL signals were dispersed by a $0.8-\mathrm{m}$ double grating monochromator and detected by a Si photodiode. In time-resolved DEPL experiments, an optical chopper and/or a liquid crystal retarder were used for the amplitude modulation of the excitation beams and the resulting DEPL transient signals were recorded by a digital oscilloscope. ODMR measurements were performed at $5 \mathrm{~K}$ at a microwave (MW) frequency of $9.21 \mathrm{GHz}$ (X-band). The $532 \mathrm{~nm}$ line of a solid-state laser was used as an excitation source. ODMR signals, which represent MW-induced changes of the PL intensity, ${ }^{31}$ were detected by a Si detector when monitoring the PL emissions within the visible spectral range of $570-810 \mathrm{~nm}$.

\section{RESULTS AND DISCUSSION}

\section{A. PL enhancement induced by the secondary NIR excitation and its origin}

Under the above-band-gap optical excitation (P1) alone, all studied $\mathrm{Ga}$ (In)NP structures exhibit strong PL emissions in the visible spectral range (the dashed lines in Fig. 2(a)), known to arise from recombination of excitons bound at N-related localized states and their phonon replica. ${ }^{32-34}$ Additional optical excitation with below-bandgap photons $(\mathrm{P} 2)$ results in a strong increase in the intensity of these PL emissions in the MBE-grown structures (the solid lines in Fig. 2(a)).

TABLE I. List of the Ga(In)NP samples studied in this work, with the main growth parameters and the hydrogen dose used in the post-growth hydrogenation. The numbers 2.5 and 18 in the parenthesis refer to the thicknesses of the $\mathrm{Ga}_{0.45} \mathrm{In}_{0.55} \mathrm{~N}_{0.016} \mathrm{P}_{0.984} \mathrm{QW}$ and GaN ${ }_{0.02} \mathrm{P}_{0.98}$ barrier layers in the MQW structure. The maximum values of the PL enhancement factor Q induced by the excitation with below-bandgap light are given in the last row.

\begin{tabular}{|c|c|c|c|c|c|c|c|c|c|c|c|}
\hline \multirow{3}{*}{$\begin{array}{l}\text { Series } \\
\text { Sample No. } \\
\text { Growth method }\end{array}$} & \multicolumn{2}{|c|}{ (1) $\mathrm{GaNP} / \mathrm{GaP}$} & \multicolumn{2}{|c|}{ (2) GaNP:H/GaP } & \multicolumn{2}{|c|}{ (3) $\mathrm{GaInNP} / \mathrm{GaP}$} & \multicolumn{2}{|c|}{ (4) GaNP:(Mg,S)/GaP } & \multicolumn{2}{|c|}{ (5) $\mathrm{GaNP} / \mathrm{Si}$} & \multirow{2}{*}{$\begin{array}{c}\text { (6) VPE-GaNP/GaP } \\
\# 15\end{array}$} \\
\hline & \#2666 & \#L012 & $\# 2666+\mathrm{H}$ & \#L012+H & \#L034 & \#L0310 & \#L037 & \#L038 & L001 & S200 & \\
\hline & GS-MBE & SS-MBE & GS-MBE & SS-MBE & \multicolumn{2}{|c|}{ SS-MBE } & \multicolumn{2}{|c|}{ SS-MBE } & SS-MBE & GS-MBE & VPE \\
\hline Growth T $\left({ }^{\circ} \mathrm{C}\right)$ & 520 & 590 & 520 & 590 & 460 & 590 & 590 & 590 & 420 & 564 & $610-650$ \\
\hline$[\mathrm{N}](\%)$ & 0.05 & 1.4 & 0.05 & 1.4 & 1.8 & $1.6 / 2$ & 0.6 & 0.6 & 1.8 & 1 & 0.6 \\
\hline$[\operatorname{In}](\%)$ & $\ldots$ & $\ldots$ & $\ldots$ & $\ldots$ & 5 & $55 / 0$ & $\ldots$ & $\ldots$ & $\ldots$ & $\ldots$ & $\ldots$ \\
\hline Structure & epi & epi & epi & epi & epi & MQW & epi & epi & epi & NWs & epi \\
\hline Thickness (nm) & 250 & 100 & 250 & 100 & 100 & $(2.5 / 18) \times 3$ & 100 & 100 & 1500 & 1000 & 100 \\
\hline $\mathrm{GaP}$ cap $(\mathrm{nm})$ & $\ldots$ & 100 & $\ldots$ & 100 & 20 & 20 & 20 & 20 & $\ldots$ & $\ldots$ & 200 \\
\hline $\begin{array}{l}\text { Shallow doping } \\
\left(10^{18} \mathrm{~cm}^{-3}\right)\end{array}$ & $\cdots$ & $\cdots$ & $\cdots$ & $\cdots$ & $\cdots$ & $\cdots$ & p-type $\mathrm{Mg}: 1.2$ & n-type $S: 1.8$ & $\cdots$ & $\cdots$ & $\cdots$ \\
\hline $\begin{array}{l}\text { Post-growth H dose } \\
\left(10^{18} \mathrm{~cm}^{-3}\right)\end{array}$ & $\cdots$ & $\cdots$ & 1 & 1 & $\cdots$ & $\cdots$ & $\cdots$ & $\cdots$ & $\cdots$ & $\cdots$ & $\cdots$ \\
\hline $\mathrm{Q}(\%)$ & 20 & 20 & 16 & 7 & 25 & 22 & 17 & 2 & 0 & 2 & 0 \\
\hline
\end{tabular}




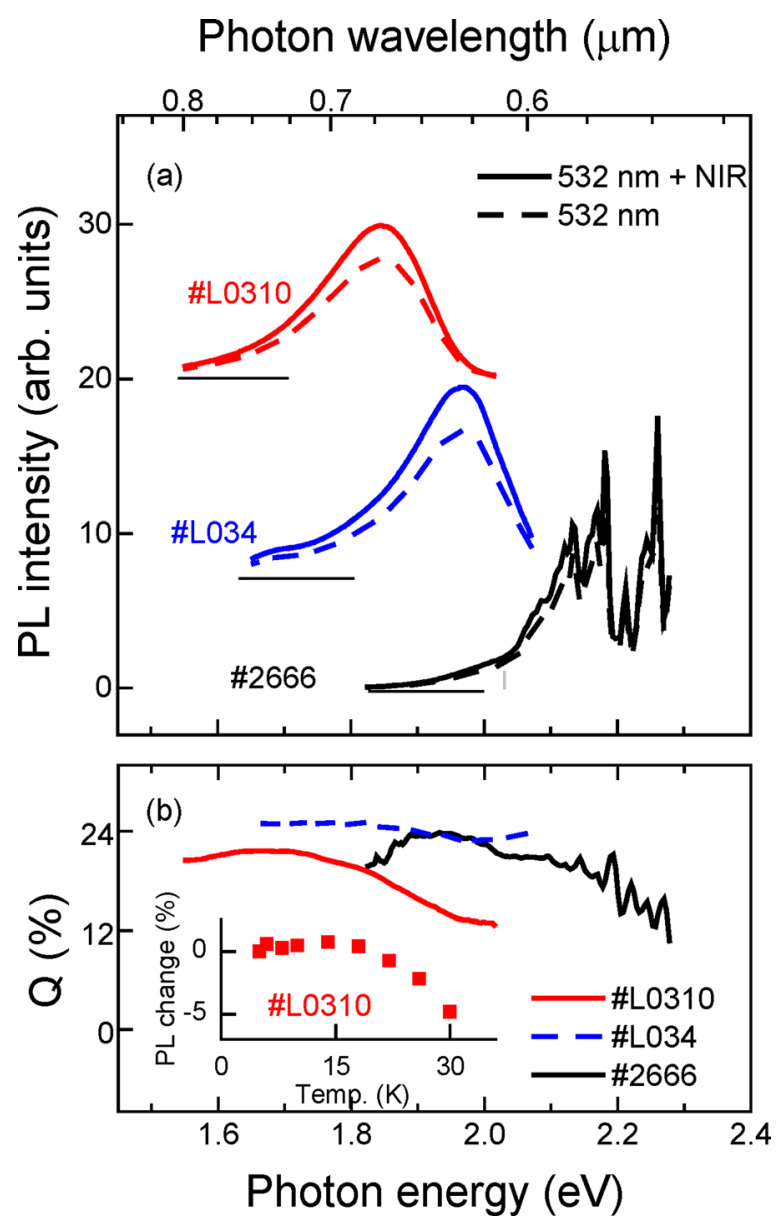

FIG. 2. (a) Representative PL spectra from three different structures containing the $\mathrm{Ga}$ (In)NP alloys, obtained at $5 \mathrm{~K}$ under the 532-nm excitation above the bandgap energies (the dashed curves) and under the dual excitation (the solid curves) of both above and below bandgap. (b) The P2-induced enhancement factor Q of the PL emissions shown in (a). The inset shows the evolution of the PL intensity with increasing temperature for the sample \#L0310. The power of the below-bandgap excitation P2 was $95 \mathrm{~mW}$ for the $0.8-1.0 \mathrm{eV}$ light or $170 \mathrm{~mW}$ for the $1.53 \mathrm{eV}$ light. The power of P1 (the 532$\mathrm{nm}$ excitation) was $5 \mathrm{~mW}$.

The PL intensity under the dual excitation, $\mathrm{PL}_{\mathrm{P} 1+\mathrm{P} 2}$, was up to $25 \%$ higher compared to the PL intensity observed under the $\mathrm{P} 1$ excitation alone, $\mathrm{PL}_{\mathrm{P} 1}$. The magnitude of this effect will be described below by a factor $\mathrm{Q}$ in percentages of the PL enhancement, defined by

$$
Q=100 *\left(\frac{\mathrm{PL}_{\mathrm{P} 1+\mathrm{P} 2}}{\mathrm{PL}_{\mathrm{P} 1}}-1\right) .
$$

From the spectral dependence of Q displayed in Fig. 2(b), the PL enhancement occurs for the entire PL emissions with a somewhat larger $\mathrm{Q}$ for the deeply localized $\mathrm{N}$ states on the low energy side.

There are in principle several possible physical mechanisms that could lead to this PL enhancement. The first possibility is that P2 with a below-bandgap photon energy could generate free electrons and holes via defect-mediated upconversion or two-photon nonlinear processes, ${ }^{35,36}$ leading to enhanced PL emissions. This possibility is not plausible here because photo-excitation with only the NIR light P2 did not result in any sizable PL emissions. The second possibility is that the excitation P2 merely causes an increase in lattice temperature, which in turn affects the PL intensity as it is usually sensitive to temperature. To test this scenario, we studied thermally-induced changes of the PL intensity under the P1 excitation alone. As it can be seen from the inset in Fig. 2(b), the intensity of the monitored PL emissions monotonously decreases with increasing temperature. This safely rules out heating induced by the $\mathrm{P} 2$ excitation as the main mechanism for the PL enhancement in the DEPL experiments. We can also exclude the possibility that the observed enhancement was due to increased carrier feeding from the $\mathrm{GaP}$ buffer layer or GaP substrate. This is based on the observed significant difference in $\mathrm{Q}$ factors between the $\mathrm{p}$ and n-type doped GaNP/GaP structures, despite the fact the $\mathrm{GaP}$ buffer layer and substrate of the two structures (the 4th pair of samples in Table I) are identical. This conclusion is further supported by our finding that the P2-induced increase in the PL intensity is also observed when the energy of the $\mathrm{P} 1$ excitation is below the bandgap of GaP, e.g., by using the $632.8 \mathrm{~nm}$ line of a He-Ne laser for excitation of the $\mathrm{Ga}_{0.45} \mathrm{In}_{0.55} \mathrm{~N}_{0.016} \mathrm{P}_{0.984} / \mathrm{GaN}_{0.02} \mathrm{P}_{0.98} \quad \mathrm{MQW}$ (not shown here). These experimental findings imply that the process enhancing the PL intensity must take place inside the $\mathrm{Ga}$ (In)NP alloys, which is not related to lattice heating by P2. A possible effect of surface depletion (leading to a socalled PL dead layer) that may be altered by NIR excitation can also be safely ruled out because the NIR-induced PL enhancement was observed in the samples with or without a GaP cap layer, as demonstrated by the first pair of the samples given in Table I. This leads us to the most plausible explanation for the observed PL enhancement induced by the secondary NIR excitation, that is, photo-excitation of carriers trapped at defect levels within the Ga(In)NP bandgap.

\section{B. The photo-excitation threshold of the PL enhancement: Energy level positions of the traps}

In order to determine the energy level positions of the carrier traps responsible for the observed strong PL enhancement under the secondary light excitation P2, we measured $\mathrm{Q}$ as a function of photon energy of $\mathrm{P} 2$. The results from two different $\mathrm{Ga}(\mathrm{In}) \mathrm{NP}$ epilayers are shown in Fig. 3. Two threshold energies, $\mathrm{E}_{\mathrm{A}}=0.56$ and $\mathrm{E}_{\mathrm{B}}=0.78 \mathrm{eV}$, which correspond to the energy positions of the relevant defect levels from either the VB edge or the CB edge, are clearly seen and are identical for both samples. This is despite the fact that these two Ga(In)NP epilayers exhibit a large difference in their bandgap energies, evident from the observed large difference in the energies of their PL emissions shown in Fig. 2(a), which is known to be mainly due to their different magnitudes of the $\mathrm{CB}$ downshift as a result of different $\mathrm{N}$ compositions. Therefore, the observed identical threshold energies $\mathrm{E}_{\mathrm{A}}$ and $\mathrm{E}_{\mathrm{B}}$ in these two alloys imply that the corresponding photo-excitation processes occur between the two deeplevels and the VB. In other words, there exist two hole traps with their energy levels located at 0.56 and $0.78 \mathrm{eV}$ above the VB edge as illustrated in Fig. 1. It should be pointed out that even though $E_{A}$ and $E_{B}$ have identical values for these two alloys, the absolute values of $\mathrm{Q}$ are different between 


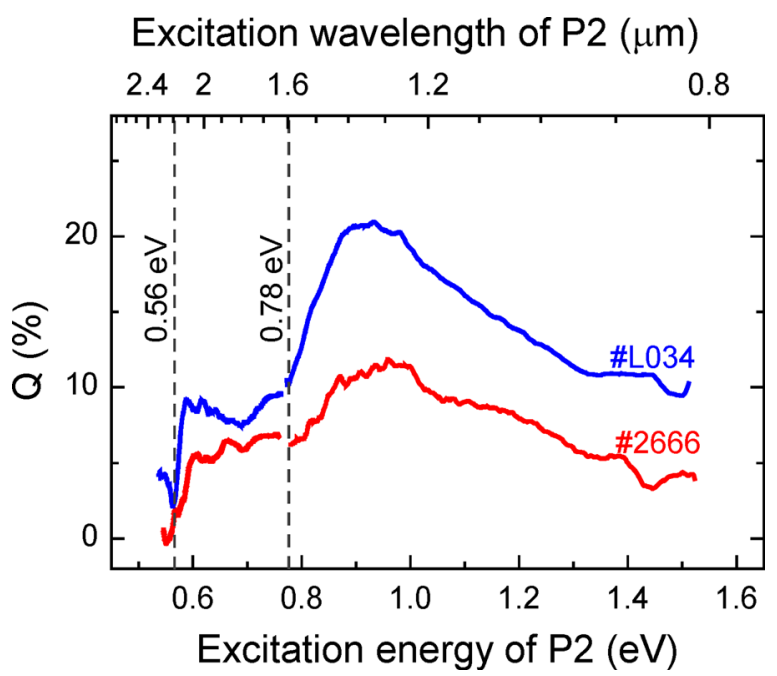

FIG. 3. DEPL excitation spectra of two Ga(In)NP alloys, obtained at $5 \mathrm{~K}$ by monitoring at $627 \mathrm{~nm}$ (for the sample \#L034) and $592 \mathrm{~nm}$ (for the sample \#2666) of the PL emissions. The photo-excitation threshold energies are marked by the vertical dashed lines. The $532 \mathrm{~nm}$ laser line was used as P1 with a power of $2 \mathrm{~mW}$. The power range of $\mathrm{P} 2$ was about $1-15 \mathrm{~mW}$.

them. As the hole de-trapping rate scales with the trapped hole concentration at a given trap level, the different $\mathrm{Q}$ values observed in these two alloys could be taken as an indication for their different concentrations of the hole traps. It is also interesting to note that the ratio between the $\mathrm{Q}$ values after reaching each of the two threshold energies is rather similar in these two alloys. This may imply a similar ratio in the product of the photo-excitation cross-section and trap density between the two traps in both alloys. Although this could merely be a coincidence, it could also indicate that the traps in question might have the same origin or are somehow linked to each other as will be further discussed below in connection with possible origins of the defects responsible for the hole traps.

\section{Excitation power dependence}

The observed PL enhancement by de-trapping of holes from defects is determined by the difference in the steadystate concentrations of VB holes between simultaneous $\mathrm{P} 1$ and $\mathrm{P} 2$ excitation and P1 excitation alone. It is governed by a detailed balance in the hole trapping and P2-induced de-trapping processes. The hole trapping scales with the number of VB holes that are available to be trapped and the concentration of the unoccupied hole traps that can receive holes from the VB. On the other hand, the P2-induced detrapping should be proportional to the optical cross-section that is specific for a given trap, the density of optical excitation P2 that releases carriers from the trap, and the concentration of trapped holes at the defect. An increase in excitation power of $\mathrm{P} 2$ promotes de-trapping and at the same time increases trapping as the densities of VB holes and the unoccupied hole traps increase due to de-trapping. As a result, the expected dependence of $\mathrm{Q}$ on the excitation power of P2 should be highly nonlinear. In order to verify the role of the hole de-trapping processes in the studied $\mathrm{Ga}(\mathrm{In}) \mathrm{NP}$ alloys, we studied excitation power dependence of Q. This was done by measuring the $\mathrm{Q}$ factor as a function of $\mathrm{P} 2$ incident power at three different P1 excitation power levels. As shown in Fig. 4, Q is found to be a strong function of the $\mathrm{P} 2 / \mathrm{P} 1$ ratio. It first increases when $\mathrm{P} 2 / \mathrm{P} 1$ increases over three orders of magnitude within the range $\mathrm{P} 2 / \mathrm{P} 1<10$. As expected, the observed increase is nonlinear for all three excitation powers of $\mathrm{P} 1$. With a further increase in $\mathrm{P} 2 / \mathrm{P} 1$ over the range of $10<\mathrm{P} 2 / \mathrm{P} 1<100$, Q starts to saturate. This can be attributed to the situation when the re-trapping of holes from the VB to the traps has increased to such a level that it completely compensates the P2-induced de-trapping as more and more trapped holes have been released from the defects to the VB by increasing P2. The saturation $\mathrm{Q}$ value is thus determined by the maximum concentration of holes that can be photo-excited by $\mathrm{P} 2$ from the defects in the steady-state condition under both $\mathrm{P} 1$ and $\mathrm{P} 2$ excitation.

It is interesting to note that the $\mathrm{P} 2 / \mathrm{P} 1$ ratio required to reach the saturation of $\mathrm{Q}$, as well as the overall dependence of $\mathrm{Q}$ on $\mathrm{P} 2 / \mathrm{P} 1$, is nearly identical at all $\mathrm{P} 1$ values that varied by more than 20 times. We suggest that this is because the share of photo-excited holes between those participating in the monitored PL and those being trapped by the defects (depending on the defect concentrations) is nearly fixed at all excitation power values of P1 for a given sample. Stronger P1 means a higher density of the trapped holes, which subsequently requires proportionally stronger P2 to de-trap the holes from the defects to reach the same degree of PL enhancement. The saturation value of $\mathrm{Q}$ should then somewhat reflect the extent of holes that were trapped by the defects with respect to the total number of photo-generated holes by P1, which is $25 \%$ in the sample shown in Fig. 4. The saturation values are found to vary among different $\mathrm{Ga}(\mathrm{In}) \mathrm{NP}$ alloys, see Table I, which indicates different concentrations of the studied hole traps. When P2/P1 $>100$ for the sample shown in Fig. 4, Q at the lowest P1 seems to start to decrease. We suggest that this is due to increasing importance of other competing processes under the same belowbandgap excitation $\mathrm{P} 2$ when the absolute number of the

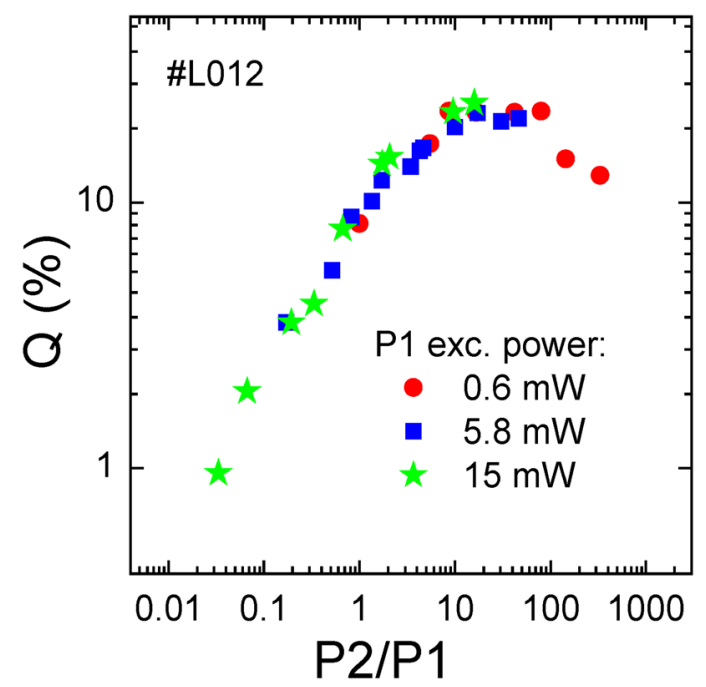

FIG. 4. (a) The measured Q values as a function of the P2/P1 ratio (the symbols) at three different $\mathrm{P} 1$ as specified, obtained at $5 \mathrm{~K}$ with a $\mathrm{P} 2$ wavelength of $870 \mathrm{~nm}$. 
trapped holes at the studied defects has significantly decreased. A plausible competing channel could be photoexcitation of the electrons from $\mathrm{N}$-related states that gives rise to the monitored PL emissions, thereby reducing the PL intensity. ${ }^{37}$

\section{Dynamics of the trapping and photo-induced de-trapping processes}

In order to shed light on the dynamics of the trapping and de-trapping processes, we carried out a detailed timeresolved DEPL study. Fig. 5 shows the measured time variations of the PL intensity under various combinations of pulsed P1 and P2 excitation. As it can clearly be seen from the region III of the experimental curve (the top curve in Fig. 5), both rise and decay of the PL intensity under the pulsed P1 excitation alone are faster than the experimental resolution of $\sim 100 \mu \mathrm{s}$. This is in agreement with our earlier time-resolved PL study of GaNP alloys by using ultrafast laser spectroscopy, from which the rise and decay times were determined to be in the range of 1 and $150 \mathrm{~ns}$, respectively. ${ }^{5}$ Equally fast rise and decay were also observed when P1 is switched on and off in the presence of cw P2 excitation, see the region I of the experimental curve in Fig. 5. All these observations can be attributed to fast photo-generation of excitons at the light-emitting N-related centers by P1 and short lifetimes of these excitons. The observed slow decrease in the PL intensity during the P1 pulse seen in region I can be explained as follows. At the instant when P1 is switched on, the number of VB holes provided by P2-induced de-trapping is determined by the steady-state value under the P2 excitation alone. This value is higher than the steady-state value under both $\mathrm{P} 1$ and $\mathrm{P} 2$ excitation (represented by the saturation value of $\mathrm{PL}_{\mathrm{P} 1+\mathrm{P} 2}$ shown in the region II of Fig. 5), as $\mathrm{P} 1$ provides more VB holes that enhance the hole trapping. As a result, the PL intensity undergoes a gradual transition between the two values during the P1 pulse, of which the transition speed is governed by the hole trapping rate that is supported by the experimental decay curve due to the hole

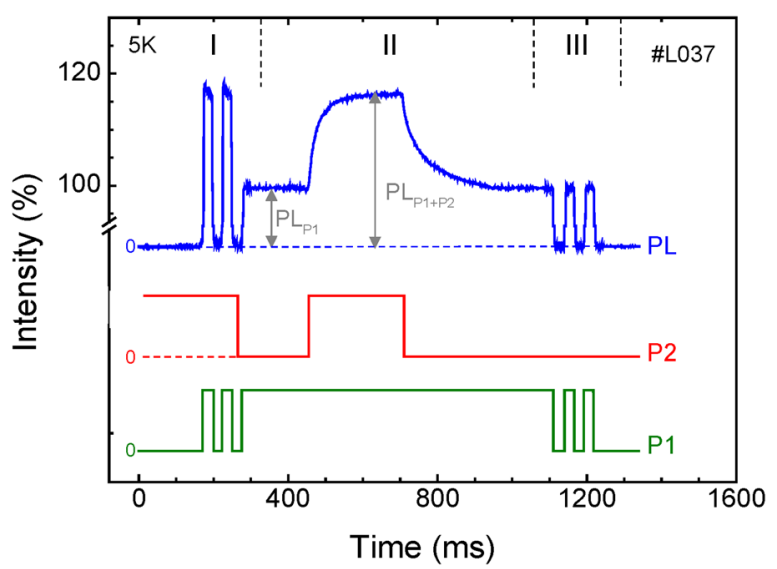

FIG. 5. The topmost curve is the time variation of the PL intensity (detected at the emission energy of $2.1 \mathrm{eV}$ ) under various combinations of the pulsed $\mathrm{P} 1$ and $\mathrm{P} 2$ excitation, measured at $5 \mathrm{~K}$ from the $\mathrm{Mg}$-doped $\mathrm{GaN}_{0.006} \mathrm{P}_{0.994}$ alloy. The lowest two curves show the pulse sequences of the P2 (at $1.355 \mathrm{eV}$ ) and $\mathrm{P} 1$ (at $2.33 \mathrm{eV}$ ) excitation beams. The PL intensities PL $\mathrm{P}_{1}$ and $\mathrm{PL}_{\mathrm{P} 1+\mathrm{P} 2}$ are defined in the figure. trapping shown in the region II of Fig. 5. We should point out that photo-excitation with only the P2 light did not result in any sizable PL emissions, as shown in the region I of Fig. 5, confirming our results from the cw PL experiments presented above.

When the samples were exposed to a constant P1 excitation, an excitation pulse of $\mathrm{P} 2$ yields a noticeably slower rise and decay of the PL intensity as seen from the region II of Fig. 5. The observed rise of the PL intensity from $\mathrm{PL}_{\mathrm{P} 1}$ to $\mathrm{PL}_{\mathrm{P} 1+\mathrm{P} 2}$ should directly reflect the dynamics of the hole detrapping when P2 is switched on, whereas the fall from $\mathrm{PL}_{\mathrm{P} 1+\mathrm{P} 2}$ back to $\mathrm{PL}_{\mathrm{P} 1}$ when $\mathrm{P} 2$ is switched off stems from the hole re-trapping by the defects. Unlike that under abovebandgap excitation P1, the rise and decay of the PL intensity due to an additional below-bandgap excitation pulse P2 occur in the range of milliseconds to tens of milliseconds, which are about five to six orders of magnitude slower than that observed under only the pulsed P1 excitation. The observed decay dynamics is identical to the PL decay during the P1 pulse in the region I of Fig. 5, supporting our conclusion that both are related to the hole trapping process.

To understand the detailed dynamics of the hole detrapping and re-trapping processes, we studied the rise and decay of the PL intensity as a function of powers of both P1 and $\mathrm{P} 2$ excitation. The results are shown in Fig. 6. The rise can approximately be described by a simple single exponential function, shown by the solid curves in Figs. 6(a) and $6(\mathrm{~b})$. The determined rise time is found to be nearly unaffected by the P1 excitation power (Figs. 6(a) and 6(c)). In sharp contrast, it speeds up at higher excitation powers of the below-gap excitation P2 (Figs. 6(b) and 6(d)), approximately as a function of $\mathrm{P} 2^{-0.5}$. The de-trapping rate of holes from
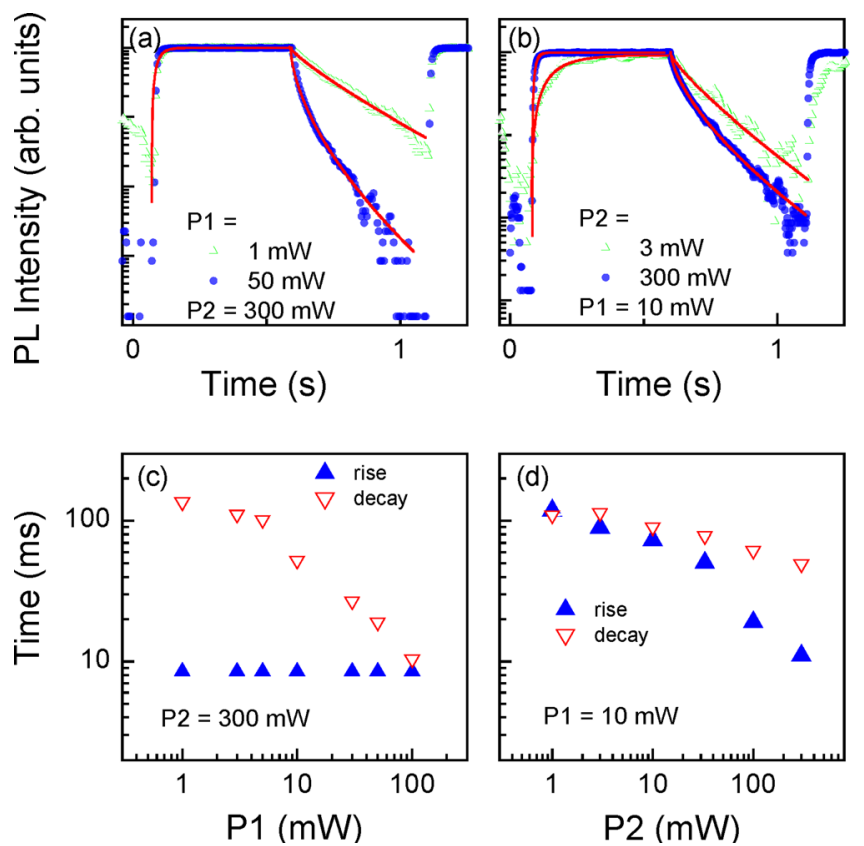

FIG. 6. (a) and (b) Typical temporal evolutions of the PL emission when the $\mathrm{P} 2$ excitation $(915 \mathrm{~nm})$ is switched on at $\mathrm{t} \sim 0.07 \mathrm{~s}$ and switched off at $\mathrm{t} \sim 0.6 \mathrm{~s}$. The solid lines are the fittings of the PL rise (by a single exponential function) and decay transients (by a stretched exponential function). The rise and decay time constants are displayed as a function of (c) P1 and (d) P2. All measurements are done on the Mg-doped GaNP structure (\#L037). 
the defects by $\mathrm{P} 2$, which is inverse to the rise time, should be directly proportional to the photon flux of the excitation light P2 and the density of the trapped holes. This explains the observed strong effect of $\mathrm{P} 2$ on the rise time. P1 can indirectly affect the rise time as it plays a key role in the number of photo-excited holes that are subject to trapping by the defects. In contrast to the rising, the decay caused by the hole re-trapping clearly does not follow a simple exponential law, a rather common feature for earlier persistent photoconductivity and DEPL experiments in other semiconductors. ${ }^{38}$ This can be understood by the fact that the re-trapping rate scales with the density of the empty traps, which continuously decreases during the hole re-trapping process. To obtain an approximate quantitative description of the decay time such that it can be compared under different excitation conditions, we modeled the decay by assuming a stretchedexponential function

$$
P L(t)=\left(P L_{P 1+P 2}-P L_{P 1}\right) e^{-\left(\frac{t}{\tau}\right)^{b}} .
$$

Here, $\tau$ is the DEPL decay time, and $b$ is the stretching exponent, with $0.48<\mathrm{b}<1$ reflecting dispersive properties of the trapping rates. As shown in Fig. 6, the decay accelerates by increasing $\mathrm{P} 1$ and $\mathrm{P} 2$ excitation power. It has a somewhat stronger dependence on P1, however. This is consistent with the fact that the density of the empty traps is determined by both the P1 and P2, namely, the former determines the number of defects available to trap holes and the latter decides how many of these available traps are empty.

\section{E. Temperature dependence}

The effect of lattice temperature on the observed PL enhancement due to hole de-trapping was investigated by measuring the $\mathrm{Q}$ values as a function of sample temperatures ranging from 5 to $160 \mathrm{~K}$, as shown in Fig. 7(a) for two different GaNP alloys. Q was found to be nearly constant below $100 \mathrm{~K}$, followed by a rapid decrease at temperatures above $100 \mathrm{~K}$. Above $160 \mathrm{~K}$, Q became negligibly small. We suggest that this is probably caused by a thermally activated acceleration of the hole trapping and thermal emission of holes from the traps, which are known for other semiconductors to exponentially increase with increasing temperature. These
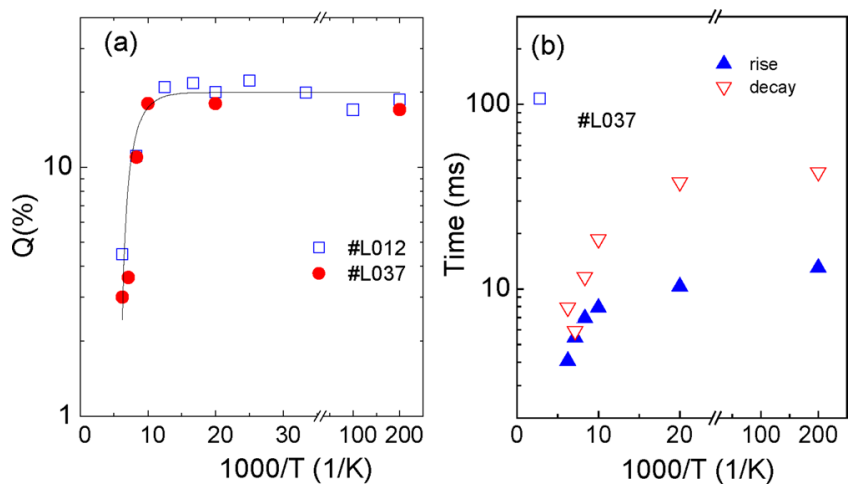

FIG. 7. Temperature dependence of (a) the Q values and (b) the rise and decay times. The solid line is just a guide to the eye. The power of P1 and $\mathrm{P} 2$ was 50 and $300 \mathrm{~mW}$, respectively. processes could eventually become more efficient than the P2-induced de-trapping process, thereby overshadowing the effect of the latter. This suggestion seems to be consistent with a drastic shortening of the hole trapping time (i.e., the decay time) over the sample temperature range, see Fig. 7(b), which is much more pronounced as compared with the thermal variation of the P2-induced de-trapping time. We cannot completely rule out other processes that could also possibly contribute to the observed decrease in the Q factor with rising temperature. One of such processes is thermally activated emission of electrons from the $\mathrm{N}$-centers to the conduction band where they become more mobile at high temperatures and could recombine with the holes trapped at the defects.

\section{F. Formation and possible nature of the hole traps}

In an attempt to understand formation and possible chemical identity of the defects responsible for the observed hole traps, effects of growth temperature, alloying with $\mathrm{N}$ and In, shallow doping, and post-growth treatments on $\mathrm{Q}$ were studied and are summarized in Table I. No clear correlation was found between $\mathrm{Q}$ and growth temperature, the $\mathrm{N}$ and In compositions, or strain, even for the highly strained $\mathrm{Ga}_{0.45} \mathrm{In}_{0.55} \mathrm{~N}_{0.016} \mathrm{P}_{0.984} / \mathrm{GaN}_{0.02} \mathrm{P}_{0.98} \mathrm{MQW}$ structure. This implies that $\mathrm{Q}$ is largely insensitive to these growth and material parameters, indicating that the observed traps are common grown-in defects in these MBE-grown Ga(In)NP alloys.

Post-growth treatments, such as rapid thermal annealing (RTA) and post-growth hydrogenation, are commonly used to passivate various defects and traps in semiconductors. Both RTA $\left(1 \mathrm{~min}\right.$ at $\left.700{ }^{\circ} \mathrm{C}\right)$ and post-growth $\mathrm{H}$ treatments were found to reduce the $\mathrm{Q}$ values, but they do not completely quench the effect of the PL enhancement (see Table I). The difference in the degree of reduction of $\mathrm{Q}$ between the two hydrogenated samples is due to their difference in the extent of hydrogen passivation of the hole traps. This could be attributed to consumption of hydrogen by other defects due to formation of $\mathrm{H}$-defect complexes, to an extent depending on the types and concentrations of these defects in each sample. Since the two hydrogenated samples were grown at different temperatures, with different $\mathrm{N}$ compositions, and by different MBE methods, namely, solid-source MBE vs. gas-source MBE, grown-in defects or residual impurities in these two samples are likely different in terms of both types and concentrations. On the other hand, Q was negligibly small in the structures which were either (i) ntype doped or (ii) grown on Si substrates (see Table I and Fig. 8). The presence of shallow n-type dopants could influence the studied PL enhancement effect by charge compensation via electron transfer ${ }^{39}$ that annihilates with the holes trapped at the defects. By annihilating electron-hole pairs, this transfer process also acts as a competing non-radiative recombination channel ${ }^{39}$ that could provide an explanation for the observed weaker PL intensity in the n-type sample. For the structures grown on Si substrates, on the other hand, the GaNP alloys could possibly be unintentionally n-type doped as a result of diffusion of $\mathrm{Si}$ atoms from the substrate. In view of charge compensation, the p-type doping by 

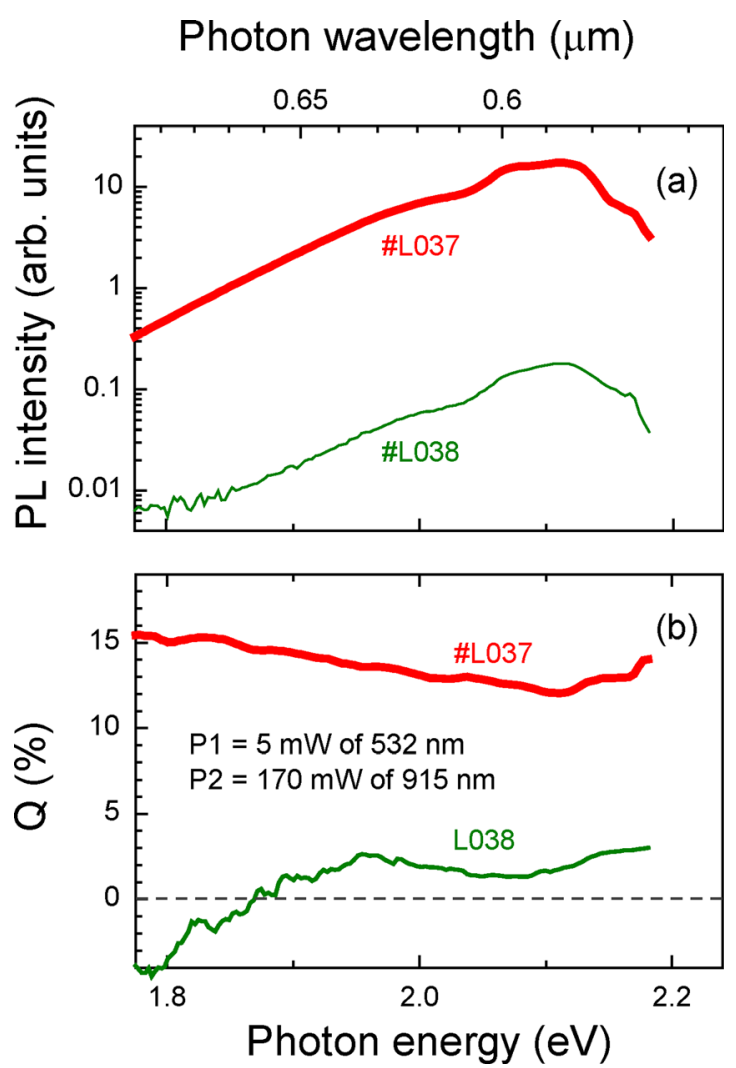

FIG. 8. (a) Representative PL spectra taken at $5 \mathrm{~K}$ from the $\mathrm{GaN}_{0.006} \mathrm{P}_{0.994}$ alloys doped with either $\mathrm{S}$ (the thin line) or $\mathrm{Mg}$ (the thick line). (b) Spectral dependence of the $\mathrm{Q}$ factors for the two samples.

shallow acceptors should not destroy the PL enhancement effect induced by the hole traps. On the contrary, it may even promote the effect as it can increase the number of holes at the traps via hole transfer from the shallow acceptors.

In order to shed light on possible defects present in the alloys, we resorted to magnetic resonance techniques, in particular, the ODMR technique that has the required sensitivity for studies of defects in thin films and quantum structures. $^{27,31,40,41}$ Previous ODMR studies ${ }^{9,17-22}$ have shown that two different species of Ga self-interstitials $\left(\mathrm{Ga}_{\mathrm{i}}-\mathrm{A}\right.$ and $\left.\mathrm{Ga}_{\mathrm{i}}-\mathrm{B}\right)$ are commonly found in $\mathrm{Ga}(\mathrm{In}) \mathrm{NP}$, and dominate in their ODMR spectra. If the studied traps give rise to an ODMR signal in one of the charge states before or after the P2-induced excitation of the trapped holes, a change in their ODMR spectra would be observed when the P2 excitation is added to the primary $\mathrm{P} 1$ excitation. This hypothesis was tested and the results are depicted in Fig. 9, for a representative case of the $\mathrm{Ga}_{0.45} \mathrm{In}_{0.55} \mathrm{~N}_{0.016} \mathrm{P}_{0.984} / \mathrm{GaN}_{0.02} \mathrm{P}_{0.98}$ MQW structure. Unfortunately, no difference in the ODMR spectra was observed upon additional P2 excitation. This finding indicates that neither $\mathrm{Ga}_{\mathrm{i}}-\mathrm{A}$ nor $\mathrm{Ga}_{\mathrm{i}}-\mathrm{B}$ is taking part in the studied hole trapping and de-trapping processes. It also implies that the trapping, photo-induced de-trapping, and recombination processes associated with the hole traps are not spin dependent, such that the involved defects eluded detection by ODMR.

Though no positive experimental identification on the origin of the studied hole traps can be obtained in the present study, a comparison of our results with reported defects in

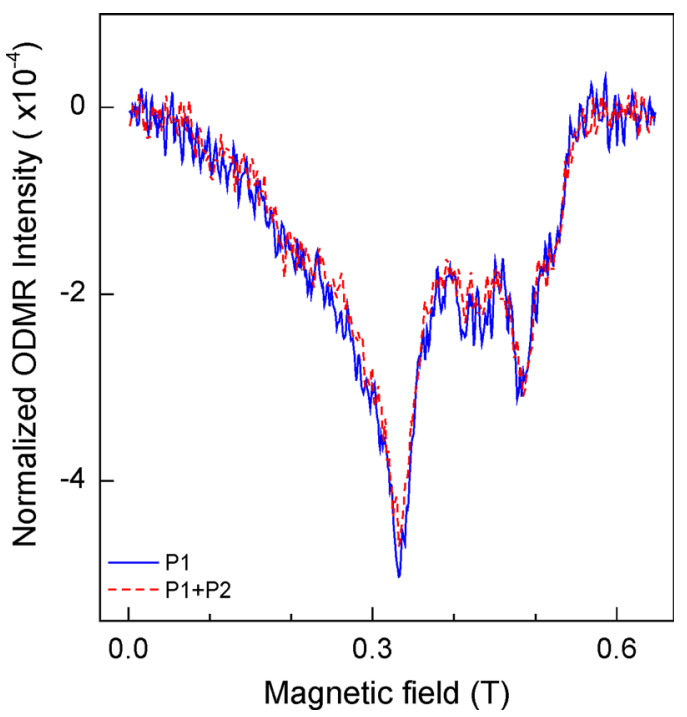

FIG. 9. ODMR spectra from the $\mathrm{Ga}_{0.45} \mathrm{In}_{0.55} \mathrm{~N}_{0.016} \mathrm{P}_{0.984} / \mathrm{GaN}_{0.02} \mathrm{P}_{0.98} \mathrm{MQW}$ structure, obtained by detecting the visible PL and using only above bandgap excitation P1 with the wavelength of $532 \mathrm{~nm}$ (the solid curve) and the dual excitation with 532 and $870 \mathrm{~nm}$ (the dashed curve). The ODMR intensity is normalized by the PL intensity.

the literature yielded useful information on possible candidates. Several traps have been observed and identified in GaP:N. ${ }^{42} \mathrm{~N}$-related traps are commonly observed with photo-ionization energies in the range of $0.36-0.46 \mathrm{eV}$. It should be noted that the nitrogen-related defects are known to give rise to electron traps. Moreover, their photoionization energies greatly deviate from the values of $0.56 \mathrm{eV}$ and $0.78 \mathrm{eV}$ determined for the hole traps in our case. The N-related centers can therefore be excluded here. Oxygen in $\mathrm{GaP}$, substituting phosphorus in the group- $\mathrm{V}$ sublattice, yields two deep-levels with the bandgap. ${ }^{42}$ Though the absolute values of their photo-ionization threshold energies are close to that of the studied hole traps, the $\mathrm{O}_{\mathrm{P}}$ donor is a deep donor in $\mathrm{GaP}$ yielding two electron trap levels of which the photo-ionization threshold energies are measured from the CB. Therefore oxygen can also be ruled out. This conclusion is further justified by secondary ion mass spectroscopy (SIMS) of the studied structures, which shows that the concentration of $\mathrm{O}$ in the \#2666 sample is two orders of magnitude higher than that in the rest of the samples, which is not correlated with the observed $Q$ values.

In copper-diffused $\mathrm{GaP}, \mathrm{Cu}$ introduces two levels in the $\mathrm{GaP}$ band gap, namely, the B level with the energy of $\sim 0.7 \mathrm{eV}$ and the A level with the energy of $\sim 0.5 \mathrm{eV}$ relative to the $V B$ top.$^{43}$ These energy values match well with the optical threshold energies of the hole traps in our studied $\mathrm{Ga}(\mathrm{In}) \mathrm{NP}$ alloys. In addition, the total charge in the VB associated with the trap A and trap B was found to be roughly the same. ${ }^{42}$ This is in line with the observation of a similar increase in Q after reaching each of the two optical thresholds, i.e., about the same number of carriers is photo-ionized from each trap. Furthermore, doping with $\mathrm{S}$ shallow donors was observed to reduce the photo-ionization of the trap B by one order of magnitude, in line with our observation of a reduced Q-value upon doping with S. Thus, based on the observed stark similarities between $\mathrm{Cu}$ in $\mathrm{GaP}$ and the hole 
traps in our studied $\mathrm{Ga}(\mathrm{In}) \mathrm{NP}$ alloys in (i) the two hole trap levels, (ii) the photo-excitation threshold energies, (iii) a fixed ratio of photo-excited holes from the traps, and (iv) suppressed photo-excitation of the trapped holes upon doping with $\mathrm{S}$, the observed traps are tentatively assigned to a defect center involving copper.

Though $\mathrm{Cu}$ impurities are not commonly observed in MBE-grown $\mathrm{N}$-free $\mathrm{GaP}$, they have been reported in MBEgrown GaNAs in concentrations $>5 \times 10^{15} / \mathrm{cm}^{3} .44$ Based on a depth-resolved distribution of these hole traps, the introduction of $\mathrm{Cu}$ impurities was shown to be connected with the operation of the nitrogen plasma cell during GaNAs growth. We believe that this could apply as well to our case of the MBE-grown Ga(In)NP alloys. This suggestion is supported by a parallel study of a GaNP epilayer grown by VPE that did not involve an $\mathrm{N}$ plasma source, where no PL enhancement was observed (see Table I). We should point out that this VPE sample is undoped, similar to the MBE-grown undoped GaNP/GaP samples (the first pair in Table I). The observed stark contrast between the VPE and MBE samples in the $\mathrm{Q}$ value can therefore be taken as clear evidence for the absence of the hole traps in the former.

\section{CONCLUSION}

To summarize, we have shown that a strong enhancement (up to $25 \%$ ) of the PL emissions in the visible spectral range is observed in the MBE-grown $\mathrm{Ga}(\mathrm{In}) \mathrm{NP} / \mathrm{GaP}$ alloys upon illumination by a secondary NIR light below the bandgap energy of the alloys. Based on a combined study of DEPL, DEPL excitation, time-resolved DEPL and ODMR, we uncovered two deep-level hole traps at 0.56 and $0.78 \mathrm{eV}$ above the VB top to be responsible for the observed NIRinduced PL enhancement of the Ga(In)NP alloys. Our timeresolved DEPL studies revealed that the hole trapping and NIR-induced de-trapping processes occur on the time scale of $\sim$ tens of milliseconds. The observed traps were shown to be common in both gas-source and solid-source MBE-grown $\mathrm{Ga}$ (In)NP alloys, insensitive to growth temperatures ranging from $460^{\circ} \mathrm{C}$ to $590^{\circ} \mathrm{C}, \mathrm{N}$ and In compositions and strain. Based on many similarities between the studied hole traps and the $\mathrm{Cu}$ impurity in $\mathrm{GaP}$, we suggest $\mathrm{Cu}$ as a possible origin of the observed traps that originate from contamination by the nitrogen plasma source employed in the MBE growth.

\section{ACKNOWLEDGMENTS}

We thank A. Polimeni and M. Capizzi for the postgrowth $\mathrm{H}$ treatments on two of the studied samples, and $\mathrm{A}$. Yu. Egorov for providing the reference sample grown by VPE. Financial support by the Swedish Research Council (Grant Nos. 621-2011-4254 and 621-2010-3815), the Linköping Linnaeus Initiative for Novel Functional Materials (LiLI-NFM) supported by the Swedish Research Council (Contract No. 2008-6582), and Knut and Alice Wallenberg Foundation (Dnr KAW 2006.0130) is highly appreciated.

${ }^{1}$ For a review, see Physics and Applications of Dilute Nitrides, edited by I. A. Buyanova and W. M. Chen (Taylor \& Francis, London, 2004).
${ }^{2}$ For a review, see Dilute III-V Nitride Semiconductors and Material Systems, Springer Series in Material Science Vol. 105, edited by A. Erol (Springer, Berlin, 2008).

${ }^{3}$ W. G. Bi and C. W. Tu, Appl. Phys. Lett. 69, 3710 (1996).

${ }^{4}$ W. Shan, W. Walukiewicz, K. M. Yu, J. Wu, J. W. Ager, E. E. Haller, H. P. Xin, and C. W. Tu, Appl. Phys. Lett. 76, 3251 (2000).

${ }^{5}$ I. A. Buyanova, G. Pozina, J. P. Bergman, W. M. Chen, H. P. Xin, and C. W. Tu, Appl. Phys. Lett. 81, 52 (2002).

${ }^{6}$ A. Luque, A. Martí, and C. Stanley, Nat. Photon. 6, 146 (2012).

${ }^{7}$ N. López, L. A. Reichertz, K. M. Yu, K. Campman, and W. Walukiewicz, Phys. Rev. Lett. 106, 028701 (2011).

${ }^{8}$ J. F. Geisz, R. C. Reedy, B. M. Keyes, and W. K. Metzger, J. Cryst. Growth 259, 223 (2003).

${ }^{9}$ D. Dagnelund, I. A. Buyanova, X. J. Wang, W. M. Chen, A. Utsumi, Y. Furukawa, A. Wakahara, and H. Yonezu, J. Appl. Phys. 103, 063519 (2009).

${ }^{10}$ X. J. Wang, Y. Puttisong, C. W. Tu, A. J. Ptak, V. K. Kalevich, A. Y. Egorov, L. Geelhaar, H. Riechert, W. M. Chen, and I. A. Buyanova, Appl. Phys. Lett. 95, 241904 (2009).

${ }^{11}$ I. A. Buyanova, W. M. Chen, and C. W. Tu, J. Phys: Condens. Matter 16, S3027 (2004).

${ }^{12}$ X. J. Wang, I. A. Buyanova, F. Zhao, D. Lagarde, A. Balocchi, X. Marie, C. W. Tu, J. C. Harmand, and W. M. Chen, Nature Mater. 8, 198 (2009).

${ }^{13}$ Y. Puttisong, X. J. Wang, I. A. Buyanova, L. Geelhaar, H. Riechert, A. J. Ptak, C. W. Tu, and W. M. Chen, Nat. Commun. 4, 1751 (2013).

${ }^{14}$ Y. Puttisong, X. J. Wang, I. A. Buyanova, H. Carrere, F. Zhao, A. Balocchi, X. Marie, C. W. Tu, and W. M. Chen, Appl. Phys. Lett. 96, 052104 (2010).

${ }^{15}$ Y. Puttisong, I. A. Buyanova, A. J. Ptak, C. W. Tu, L. Geelhaar, H. Riechert, and W. M. Chen, Adv. Mater. 25, 738-742 (2013).

${ }^{16}$ Y. Puttisong, X. J. Wang, I. A. Buyanova, C. W. Tu, and W. M. Chen, J. Appl. Phys. 111, 07C303 (2012).

${ }^{17}$ N. Q. Thinh, I. P. Vorona, I. A. Buyanova, W. M. Chen, S. Limpijumnong, S. B. Zhang, Y. G. Hong, C. W. Tu, A. Utsumi, Y. Furukawa, S. Moon, A. Wakahara, and H. Yonezu, Phys. Rev. B 70, 121201(R) (2004).

${ }^{18}$ N. Q. Thinh, I. P. Vorona, M. Izadifard, I. A. Buyanova, W. M. Chen, Y. G. Hong, H. P. Xin, and C. W. Tu, Appl. Phys. Lett. 85, 2827 (2004).

${ }^{19}$ I. P. Vorona, T. Mchedlidze, M. Izadifard, I. A. Buyanova, W. M. Chen, Y. G. Hong, H. P. Xin, and C. W. Tu, Appl. Phys. Lett. 86, 222110 (2005).

${ }^{20}$ D. Dagnelund, I. A. Buyanova, T. Mchedlidze, W. M. Chen, A. Utsumi, Y. Furukawa, S. Moon, A. Wakahara, and H. Yonezu, Appl. Phys. Lett. 88, 101904 (2006).

${ }^{21}$ D. Dagnelund, X. J. Wang, C. W. Tu, A. Polimeni, M. Capizzi, I. A. Buyanova, and W. M. Chen, Appl. Phys. Lett. 98, 141920 (2011).

${ }^{22}$ N. Q. Thinh, I. P. Vorona, I. A. Buyanova, W. M. Chen, S. Limpijumnong, S. B. Zhang, Y. G. Hong, H. P. Xin, C. W. Tu, A. Utsumi, Y. Furukawa, S. Moon, A. Wakahara, and H. Yonezu, Phys. Rev. B 71, 125209 (2005).

${ }^{23}$ D. Dagnelund, J. Stehr, A. Yu. Egorov, W. M. Chen, and I. A. Buyanova, Appl. Phys. Lett. 102, 021910 (2013).

${ }^{24}$ N. Q. Thinh, I. A. Buyanova, W. M. Chen, H. P. Xin, and C. W. Tu, Appl. Phys. Lett. 79, 3089 (2001).

${ }^{25}$ I. P. Vorona, T. Mchedlidze, D. Dagnelund, I. A. Buyanova, W. M. Chen, and K. Köhler, Phys. Rev. B 73, 125204 (2006).

${ }^{26}$ D. Dagnelund, I. P. Vorona, G. Nosenko, X. J. Wang, C. W. Tu, H. Yonezu, A. Polimeni, M. Capizzi, W. M. Chen, and I. A. Buyanova, J. Appl. Phys. 111, 023501 (2012).

${ }^{27}$ D. Dagnelund, I. P. Vorona, L. S. Vlasenko, X. J. Wang, A. Utsumi, Y. Furukawa, A. Wakahara, H. Yonezu, I. A. Buyanova, and W. M. Chen, Phys. Rev. B 81, 115334 (2010).

${ }^{28}$ A. Dobrovolsky, J. E. Stehr, S. L. Chen, Y. J. Kuang, S. Sukrittanon, C. W. Tu, W. M. Chen, and I. A. Buyanova, Appl. Phys. Lett. 101, 163106 (2012).

${ }^{29}$ Y. J. Kuang, S. Sukrittanon, H. Li, and C. W. Tu, Appl. Phys. Lett. 100, 053108 (2012).

${ }^{30}$ S. Sukrittanon, Y. J. Kuang, A. Dobrovolsky, W.-M. Kang, J.-S. Jang, B.J. Kim, W. M. Chen, I. A. Buyanova, and C. W. Tu, Appl. Phys. Lett. 105, 072107 (2014).

${ }^{31}$ W. M. Chen, Thin Solid Films 364, 45 (2000).

${ }^{32}$ D. G. Thomas and J. J. Hopfield, Phys. Rev. 150, 680 (1966). 
${ }^{33}$ X. Liu, S. G. Bishop, J. N. Baillargeon, and K. Y. Cheng, Appl. Phys. Lett. 63, 208 (1993).

${ }^{34}$ I. A. Buyanova, G. Yu. Rudko, W. M. Chen, H. P. Xin, and C. W. Tu, Appl. Phys. Lett. 80, 1740 (2002).

${ }^{35}$ J. E. Stehr, S. L. Chen, N. K. Reddy, C. W. Tu, W. M. Chen, and I. A. Buyanova, Adv. Funct. Mater. 24, 3760 (2014).

${ }^{36}$ A. Dobrovolsky, S. Sukrittanon, Y. Kuang, C. W. Tu, W. M. Chen, and I. A. Buyanova, Small 10, 4403-4408 (2014).

${ }^{37}$ A. Z. M. T. Islam, T. Hanaoka, K. Onabe, S. Yagi, N. Kamata, and H. Yaguchi, Appl. Phys. Express 6, 092401 (2013).

${ }^{38}$ D. Redfield and R. H. Bube, Photoinduced Defects in Semiconductors (Cambridge University Press, Cambridge, 1996), p. 39, 66, 71, 118.
${ }^{39}$ W. M. Chen, B. Monemar, E. Janzén, and J. L. Lindström, Phys. Rev. Lett. 67, 1914 (1991).

${ }^{40}$ W. M. Chen and B. Monemar, Appl. Phys. A 53, 130 (1991).

${ }^{41}$ J. E. Stehr, S. L. Chen, S. Filippov, M. Devika, N. Koteeswara Reddy, C. W. Tu, W. M. Chen, and I. A. Buyanova, Nanotechnology 24, 015701 (2013).

${ }^{42}$ S. T. Pantelides, Deep Centers in Semiconductors: A State of the Art Approach, 2nd ed. (Gordon and Breach Science Publishers, Yverdon, 1992).

${ }^{43}$ P. O. Fagerström, H. G. Grimmeiss, and H. Titze, J. Appl. Phys. 49, 3341 (1978).

${ }^{44}$ P. Krispin, S. G. Spruytte, J. S. Harris, and K. H. Ploog, J. Appl. Phys. 89, $6294(2001)$. 